\title{
LA CONFIGURACIÓN DEL PAISAJE CULTURAL DURANTE LA ALTA EDAD MEDIA (SIGLOS V-XI): CAMBIOS AMBIENTALES Y ACTIVIDAD ANTRÓPICA EN EL NOROESTE DE LA PENÍNSULA IBÉRICA
}

\author{
MANUELA COSTA-CASAIS ${ }^{(1)} \&$ JOERI KAAL ${ }^{(2)}$
}

Resumen:

\begin{abstract}
Este trabajo tiene por objetivo aportar algunos elementos para el debate sobre la reconstrucción paleoambiental, desde las disciplinas de las Ciencias de la Tierra, centrándose en el período cultural de la Alta Edad Media (siglos V-XI). El artículo se divide en tres apartados. El primero, introduce conceptos básicos sobre la evolución ambiental: ideas generales sobre la reconstrucción paleoambiental y un breve comentario sobre la actividad humana en el contexto de los paleoambientes. El segundo, muestra una aproximación metodológica a la reconstrucción desde diferentes disciplinas de las Ciencias de la Tierra. En el tercero, se comentan algunos resultados recientes obtenidos en el noroeste peninsular utilizando ejemplos de archivos que, en este caso, están representados por suelos coluviales y sus señales geoquímicas, analizando los procesos naturales y otros inducidos por la actividad antrópica, con la finalidad de ilustrar los conceptos discutidos en la primera parte. A modo de conclusión, el artículo se cierra con unas pinceladas sobre el paisaje de la Alta Edad Media en el Noroeste Peninsular, a partir de la interpretación y reconstrucción de los datos obtenidos.
\end{abstract}

Palabras clave: Reconstrucción paleoambiental, Patrimonio Natural-Cultural, Antigüedad tardía, Alta Edad Media, Galicia, NW Península Ibérica

Abstract: The configuration of the cultural landscape during the early Middle Ages $\left(\mathbf{5}^{\text {th }} \mathbf{- 1 1 ^ { \text { th } }}\right.$ centuries): Environ-
mental changes and human activity in the northwest of the Iberian Peninsula.
This paper aims to promote the discussion of some elements relating to paleoenvironmental reconstruction,
from the point of view of the Earth Sciences, focusing on the cultural period of the early Middle Ages $\left(5^{\text {th }}-11^{\text {th }}\right.$
centuries). The article is divided into three sections. In the first, the basic concepts of environmental evolution
are introduced, such as the general ideas of paleoenvironmental reconstruction and a brief commentary on
human activity in the context of paleoenvironments. The second section demonstrates a methodological ap-
proach to reconstruction from the point of view of different disciplines of Earth Sciences. In the third section,
recent results obtained in the northwest of the Iberian Peninsula are commented on, making use of samples of
colluvial soils and their geochemical signals obtained from environmental archives. An analysis is made of
both natural processes and those caused by human activity, in order to illustrate the concepts discussed in the
first part. The article concludes with some comments on the landscape of the early Middle Ages in the north-
west of the Peninsula, based on the interpretation and reconstruction of the data obtained.

Keywords: Paleoenvironmental reconstruction, Natural-Cultural Heritage, late Antiquity, early Middle Ages, Galicia, NW Iberian Peninsula

\section{INTRODUCCIÓN: EL PALEOAMBIENTE COMO PATRIMONIO NATURAL Y CULTURAL}

La puesta en valor del paleoambiente desde las Ciencias de la Tierra ofrece a los profesionales de la arqueología y a los prehistoriadores una información valiosa sobre la evolución del paisaje. Pero, ¿de qué se ocupa la reconstrucción paleoambiental? Su objetivo es analizar las evidencias almacenadas en los archivos naturales y antrópicos. Sus propiedades, interpretables en términos de reconstrucción, son conocidas como señales ambientales. Las formaciones superficiales que almacenan estas señales son los archivos ambientales, y según su origen son: naturales (hielos árticos, gla- ciares, sedimentos oceánicos y lacustres, turberas, brañas, suelos policíclicos, etc...) o antrópicos (yacimientos arqueológicos, suelos de cultivo, bancales, etc...,) (MARTíNEZ CORTIZAS 2000 a). Las señales reconocidas - geomorfológicas, sedimentarias, edáficas, bióticas, geoquímicas, etc... - pueden ser identificables a simple vista o detectables tras un análisis detallado del archivo.

Para la realización de este trabajo los archivos utilizados han sido los suelos coluviales. Los procesos desarrollados en estos suelos contribuyen a una comprensión de los yacimientos arqueológicos, debido a que actúan como un archivo en múltiples direcciones (WALKINGTON 2010): (a) dando respuesta a los

${ }^{(1)}$ Departamento de Xeografía, Facultade de Xeografía e Historia, Universidade de Santiago de Compostela, Praza da Universidae 1, 15782 Santiago de Compostela, A Coruña, España. Email: manuela.costa@usc.es

(2) Instituto de Ciencias del Patrimonio (Incipit), Consejo Superior de Investigaciones Científicas (CSIC), San Roque 2, 15704 Santiago de Compostela, Spain Email: joeri.kaal@incipit.csic.es 
procesos del sistema a través de su interacción con los factores generados en el lugar concreto donde se desarrollan, (b) reflejando los procesos del medio, tanto en sentido vertical como lateral, (c) almacenando indicadores paleoecológicos - polen, fitolitos, huesos, etc.- conviertiéndose en archivos de los cambios ambientales (LOWE \& WALKER 2006), (d) siendo geoarchivos: a pesar de mostrar rasgos morfológicos que reflejan procesos de transporte, los suelos coluviales almacenan información ambiental (LEOPOLD \& VÖLKEL 2007; TALLÓNARMADA et al. 2014), y (e) registrando el uso antrópico y la gestión del paisaje.

El principal fin de la investigación prehistórica es el yacimiento arqueológico, pero la actividad humana no se circunscribe únicamente al espacio identificado como yacimiento. Este debe entenderse como un sumidero neto de recursos del medio y su entorno como la fuente que aporta dichos recursos (bióticos y abióticos) (MARTÍNEZ CORTIZAS 2000 a, b). La obtención y manejo de los recursos por el ser humano genera impactos directos, producto de su actividad (deforestación, por ejemplo) e inducidos, desencadenados colateralmente por dicha actividad (por ejemplo, erosión de los suelos y sedimentos). También produce resíduos, productos secundarios no reutilizables. Los impactos y los residuos originados en el paisaje se materializan como huellas producidas por la actividad humana en el pasado.

El objetivo de este trabajo es aportar algunos elementos para el debate de fondo sobre la reconstrucción paleoambiental desde las disciplinas de las Ciencias de la Tierra, centrándose en el período cultural de la Antigüedad Tardía/Alta Edad Media. Para ello se hace una aproximación al contexto climático y las transformaciones del paisaje en el noroeste de la Península Ibérica entre los siglos V-XI, a partir de información bibliográfica disponible y de los resultados recientes de investigación sobre los suelos coluviales y sus señales geoquímicas, a fin de analizar los procesos naturales y los inducidos por la actividad antrópica.

\section{UNA APROXIMACIÓN METODOLÓGICA A LARECONSTRUCCIÓN PALEOAMBIENTAL}

El objetivo de las Ciencias de la Tierra aplicado a la reconstrucción paleoambiental es averiguar los procesos ocurridos y las condiciones ambientales que los han generado con la finalidad de establecer la fiabilidad de las señales y los archivos analizados. Si estos son suelos, la presencia o ausencia de señales puede ser insuficiente para entender la relación entre sus propiedades y los procesos pedogenéticos, diagenéticos y/o antropogénicos. Es necesario, por tanto, utilizar una metodología variada en la reconstrucción paleoambiental, como práctica estándar para afrontar los estudios pedoarqueológicos (WALKINGTON 2010). Los pasos a seguir para la realización de la investigación deben articularse a diversas escalas, englobando: formaciones geológicas y geomorfológicas, tipos de sedimentos y suelos que las recubren, hasta llegar a la parte de detalle del estudio, dominado por la caracterización de las propiedades de los archivos -registros invisibles (señales geoquímicas, fitolitos, polen, ...) (MARTÍNEZ CORTIZAS 2000 b; COSTA-CASAIS et al. 2012). El enfoque metodológico puede articularse en cuatro fases: recopilación bibliográfica, realización de los trabajos de campo; toma de muestras de los archivos elegidos y procedimientos analíticos (Martínez Cortizas 2000 a, b) (Fig. 1).



Fig. 1. Pasos a seguir para una aproximación a la reconstrucción paleoambiental. Modificado de Martínez Cortizas (2000 a,b)

Fig. 1. Steps followed in the multi-archive, multiproxy, approach to palaeoenvironmental reconstruction. Modified from Martínez Cortizas (2000 a,b). 
La finalidad es obtener información sobre: (a) génesis de las formaciones superficiales del área, condiciones morfodinámicas implicadas y el papel de la actividad humana en los cambios superficiales; (b) procesos erosivo/acumulativos que han tenido lugar y sus posible causas; (c) caracterización de las principales rutas edafogenéticas, los ciclos de suelos y la secuencia cronoedáfica local y su relación con la regional; (d) cambios en las comunidades vegetales y su cronología; (e) cambios climáticos; (f) usos del territorio por parte de los grupos humanos, así como sus repercusiones a escala de paisaje. Estos objetivos confluyen en la diferenciación de los procesos de cambio en épocas pasadas, su origen natural o antrópico y las interacciones y ajustes entre cambios ambientales y cambios culturales.

\section{ANTIGÜEDAD TARDÍA/ALTA EDAD MEDIA (SIGLOS V-XI AD): PROCESOS NATURALES E INDUCIDOS POR LA ACTIVIDAD HUMANA}

En la primera parte de esta sección, se expone una visión general del contexto climático que envuelve la Antigüedad tardia/Alta Edad Media en lo que hoy conocemos como Europa, la Península Ibérica y el Noroeste Peninsular, mostrando unas pinceladas de las condiciones climáticas anteriores al siglo V y posteriores al XI. En la segunda, se analiza la configuración del paisaje, ligada a los procesos naturales o inducidos - erosión, quemas, acidificación, etc.

\subsection{El contexto climático del noroeste de la Península Ibérica entre los siglos V y XI}

El mundo científico se interesa cada vez más por investigar la historia del clima, tanto a nivel general como por países o regiones. En el caso de la Península Ibérica adquiere una especial relevancia dado su carácter de auténtica "encrucijada climática" (FONT TULLOT 1988). La investigación sobre las características del clima en época medieval, su magnitud en el espacio y el tiempo, sus posibles causas y su impacto en las sociedades de la época continuará, y probablemente se expandirá, ya que este período se caracteriza por importantes cambios culturales en muchas partes del mundo (WeisS \& BRAdley 2001; Diaz \& STAHLE 2007; BÜNTGEN et al. 2011). La intención de este trabajo, es acercarse a la configuración del paisaje entre los siglos V y XI. En este apartado, al tratar el factor climático, se incrementará la cronología de estudio, debido a que va a permitir mostrar una visión del clima en una escala temporal más amplia, lo que ayudará a entender de forma evolutiva, cómo fueron las condiciones climáticas que operaron a escala del paisaje en el Noroeste Peninsular antes del siglo $\mathrm{V}$ y después del $\mathrm{X}$. Se expone una imagen general del paisaje climático Europeo, peninsular y del Noroeste Ibérico desde el Episodio Cálido Romano (2000-1500 BP) (100 BC-400 AD) hasta el Episo- dio Cálido Bajomedieval (900-650 BP) (1000-1300 AD). Entre ambos, transcurre el momento cronológico que interesa para los objetivos de este artículo, el Episodio Frío Altomedieval (400-1000 AD).

\subsubsection{EI Episodio Cálido Romano (2000- 1500BP) (100 BC-400 AD)}

En Europa, desde el 100 BC hasta aproximadamente el $400 \mathrm{AD}$, se produce una mejora térmica acompañada de una caída de las precipitaciones, coincidiendo con la época romana. Este hecho es lo que hace que se denomine como "Episodio Cálido Romano". Se produce un aumento en la frecuencia de veranos cálidos y secos, y disminuyen los inviernos extremos, haciéndose más suaves. Esta manifestación térmica fue acompañada de una lenta subida del nivel del mar, que sería del orden de un metro cada cien años, y las temperaturas medias anuales alrededor de $2^{\circ} \mathrm{C}$ mas elevadas (FONT TUlLOT 1988). Este episodio, es uno de los más cálidos de los últimos 4.000 años $\mathrm{BP} \mathrm{y}$, por comparación, el promedio de los valores del índice de temperatura relativa es de $+2,2$, por lo que las temperaturas se mantuvieron entre $2^{\circ}$ y $3^{\circ} \mathrm{C}$ por encima de las medias actuales, siendo a su vez la segunda mitad de este periodo una fase más húmeda, probablemente la más larga de las del Holoceno Final (MARTínez CORTIZAS et al. 1999 a, b).

Este episodio tuvo una gran repercusión en el litoral gallego, como así lo atestiguan las evidencias de algunos asentamientos prehistóricos que quizás se vieron afectados tanto por las mareas altas como por la excepcionalidad de eventos de temporales. Se han verificado ejemplos de facies de marisma, en cotas de marea superiores a las actuales, sobre las que se construyen salinas, como las que se han descubierto en la calle de Rosalía de Castro (ciudad de Vigo). Las dataciones radiocarbónicas realizadas permiten contextualizar el momento de su explotación, desde el siglo II BC hasta el III AD (TALLÓN-ARMADA 2012). También el nivel basal del depósito de Mirambel (Ría de Vigo) está formado por una facies tipo marisma característica de un medio transgresivo, que se correspondería con este momento (MARTíNEZ CORTIZAS et al. 1996). El estudio detallado del ciclo dunar basal, localizado sobre la marisma, muestra rasgos edafogenéticos evolucionados, que según JUNGERIUS (1990) son alcanzados en aquellas dunas revegetadas y más alejadas del área fuente. Parece que este ciclo representa la evolución de una duna colonizada por la vegetación arbórea, distante de la zona mareal. Estas ideas se ven reforzadas con los datos arqueológicos que permiten contextualizarlas a nivel cronológico, ya que sobre la duna se construye la villa galaicoromana que fue ocupada entre los siglos III y VI AD (Soto ARIAS 1994; HidAlgo CuÑarro \& SOtO ARIAS, 1995; NAVEIRO LÓPEZ \& SOTO ARIAS 1996). La fase de acumulación eólica, la 
estabilización de la duna y el comienzo de la evolución edafogenética sería anterior al siglo III AD (MARTÍNEZ CORTIZAS et al. 1996; MARTÍNEZ CORTIZAS \& COSTA-CASAIS 1997). Posiblemente la acumulación comience hacia el 3000 BP (1000 $\mathrm{BC})$ coincidiendo con una cierta mejoría climática. Estos niveles dunares no muestran una evolución edáfica importante. El segundo nivel dunar, que en Mirambel fosiliza la superficie ocupada en época romana, comenzaría a depositarse en torno al 1500 -1400 BP (500-400 AD).

\subsubsection{El Episodio Frío Altomedieval (1500-1000 BP; 400-1000 AD)}

Al calentamiento observado durante los últimos años del siglo IV le suceden variaciones térmicas a comienzos del siglo $\mathrm{V}$, seguidas por un marcado frío, que se evidencia por el avance de los glaciares escandinavos y alpinos, crecidas históricas de los ríos europeos como las del Ródano (años 563-572 y 583), inviernos rigurosos con fuertes nevadas, momentos de fuertes sequías y cuantiosas pérdidas en la agricultura (olivares e higueras en la europa meridional), hielos marinos en las costas venecianas y otros puntos de Europa (años 859. 860) (FONT TUllot 1988). En Europa central y occidental se produce una fase de transición, con inviernos fríos y las demás estaciones más secas que lluviosas, coincidiendo el siglo IX con el final del período. En la Península Ibérica, este momento cronológico estuvo marcado por grandes adversidades climáticas, destacando las sequías (FONT TULlOT 1988). En el siglo VI, se produce un cambio que supuso un descenso de las temperaturas entre $2^{\circ}$ y $2,5^{\circ} \mathrm{C}$, coincidiendo con la desintegración del Imperio Romano y la presencia de poblaciones germánicas en la Península Ibérica. Esta fase de disminución de la temperatura, fue acompañada por un paisaje deforestado. En general, la deforestación incrementa la superficie de albedo (efecto biogeofísico), que induce a una tendencia de enfriamiento general en Europa, que comienza a ser observada desde Tiempos Medievales (BROvKIN et al. 2006; GoOsse et al. 2006 a; PonGRATZ et al. 2009). Desde un punto de vista paleoclimático, el inicio del episodio frío Medieval se caracterizó por temperaturas bajas y una mayor aridez, factores que contribuyeron a dificultar los cultivos con las técnicas disponibles en este período (BlANCO-GONZÁlEZ et al. 2014). En general, y con estas condicións adversas, el paisaje europeo comenzaría a ser objeto de cambios de uso del suelo (Goudie 1993; PONGRATZ et al. 2008; KAPLAN et al. 2009).

Este período de degradación climática persistió en el Noroeste Ibérico durante un siglo, pero pronto dio paso a una recuperación térmica, con valores de temperatura semejantes a los de época romana (FONT TULLOT 1988). Esta característica es singular de la evolución climática de Galicia. Mientras que el resto de Europa estuvo inmersa en el Episodio Frío Altomedieval, en Galicia entre el 650 y el $1000 \mathrm{AD}$, se disfrutaba de unas condiciones más benignas - principalmente secas y relativamente cálidas, como reflejaría la estabilización de las dunas que colmatan la salina de Rosalía de Castro (TALLÓN-ARMADA, en esta publicación). Este hecho se explica por la peculiar situación circulatoria de la atmósfera que provocó el arrastre de masas de aire frío centroeuropeo y siberianas hacia la cuenca mediterránea, originando adversidades climáticas. Pero, también generó el acceso de masas de aire oceánico cálido del sur y del suroeste sobre Galicia, favoreciendo unas condiciones climáticas claramente contrastadas con las del resto de la península (MARTínEz CORTIZAS et al. 1999 a, b; GARCÍA et al. 2005). Este episodio explicaría por qué, a partir del siglo $\mathrm{V}$, los cultivos mediterráneos irrumpen con fuerza en el norte y centro de España y justificaría la introducción de ciertos cultivos térmicamente exigentes, para este momento en Galicia. En la ría de Muros-Noia, en el depósito de Caamaño (Porto do Son, Galicia, NW Península Ibérica), CosTA-CASAIS et al. (1994) obtuvieron dos dataciones radiocarbónicas para el suelo superficial, caracterizado como ranker atlántico, de casi 2 metros de espesor. La obtenida a 160 $\mathrm{cm}$ de la superficie, prácticamente en la base de este nivel y apoyada sobre una línea de piedras, aportó una fecha de $2.7201 \pm 40 \mathrm{BP}(\mathrm{GrN}-22280)$ (3216-2455 BP /1267-506 BC). Esta edad es indicativa de que a partir de este momento se está produciendo la llegada de abundante material erosionado del continente a sectores del litoral, lo que refleja una intensa actividad erosiva-acumulativa en las laderas del entorno, que puede relacionarse con una fuerte actividad antrópica en el paisaje.

\subsubsection{El Episodio Cálido Bajomedieval (900-650 BP; 1000-1300 AD)}

El episodio cálido Bajomedieval (MWP) o Anomalía Climática Medieval (MCA), es uno de los períodos climáticos relevantes, con temperaturas de $3,5^{\circ} \mathrm{C}$ superiores a las actuales (MARTínEZ CORTIZAS et al. 1999 b). Este episodio no fue contínuo, ni fue espacialmente sincrónico a nivel geográfico (HUGHES \& DíAZ 1994; BRADLEY et al. 2003; GoOsSE et al. 2005; GUIOT et al. 2010). En este sentido cabe señalar, como caso particular, que varios registros indican condiciones frías entre el 1050 y el 1150 AD (BÜNTGEN et al. 2006; GRUDD 2008; CORONA et al. 2010) con temperaturas similares a las observadas más tarde, durante la Pequeña Edad del Hielo (LIA, 1400 y 1850). Las condiciones europeas, generalmente suaves durante la MCA, potencialmente podrían ser la consecuencia de una anomalía positiva prolongada en forzamientos radiativos externos (BARD et al. 2000). Los períodos medievales, alrededor del 900-1000 y 
1050-1250 AD se caracterizaron por una radiación solar total alta que contribuiría al calentamiento de la superficie terrestre (MUSCHELER et al. 2007; DELAYGUE \& BARD 2010). Lagos Ibéricos muestran niveles más bajos de agua y salinidades más altas durante los siglos XI al XIII. Esta aridez del Mediterráneo occidental parece coherente con el estado positivo de la NAO (Oscilación del Atlántico Norte) durante la Anomalía Climática Medieval (ROBERTS et al. 2012). Los resultados de la investigación llevada a cabo por Goose et al. (2012) ponen de relieve la complejidad de la estructura espacial y temporal de la MCA en Europa. Esta fase cálida, se manifiesta primero, a altas latitudes como un período de retroceso de los hielos en el Atlántico norte, acompañado de un descenso en la frecuencia de temporales. En la Europa central y occidental, se comienza a notar un predominio de las situaciones ciclónicas (veranos preferentemente cálidos e inviernos fríos con pocas precipitaciones y con sequías frecuentes). El apogeo de esta fase se produce entre el 1100 y el $1300 \mathrm{AD}$, cuando las lluvias y el calentamiento general repercuten en los cultivos - en muchas partes de las Islas Británicas los cultivos se extendieron a zonas altas y el vino medieval inglés llegó a ser de gran calidad, las temperaturas medias estivales en Inglaterra alcanzaron un grado más que en el siglo XX y en Europa Central más de un grado y medio (FONT TULLOT 1988). En la Europa meridional, este período se revela con el aumento de las precipitaciones y en España, el siglo XI mantiene la tónica seca de los siglos precedentes. En el siglo XII se presenta la fase lluviosa y cálida en la Península Ibérica, en toda la región mediterránea e incluso en el norte de África, donde es visible la recuperación de la vegetación y un marcado retroceso del desierto. Este fue un período bastante húmedo en los sectores atlánticos, con frecuentes riadas e inundaciones desbordamiento del Miño en 1102 AD y no hay noticias de calores excepcionales, aunque es interesante destacar la noticia del largo verano de 1190 $\mathrm{AD}$ que en Galicia se prolongó hasta mediados de enero del año siguiente (Font Tullot 1988). El siglo XIII mantiene las mismas características climáticas que el anterior, pero aún más acentuadas en relación a la pluviosidad y en Europa, el cambio hacia una nueva fase fría se comienza a manifestar después del siglo XIV (Pequeña Edad del Hielo -LIA; 1400-1800 CE) (TROUET et al. 2012).

Estudios polínicos realizados en el Sistema Central español muestran el comienzo de los impactos al medio ambiente a largo plazo en este periodo (BLANCO-GONZÁLEZ et al. 2014). Este trabajo refleja que, en cuanto a la situación del clima, este período se caracteriza por temperaturas similares a las actuales y lluvias moderadas, con una fase seca entre los siglos IX y XI. Los episodios de sequías severas han sido documentados a lo largo del periodo en la ladera sur de del Sistema Central.

En un contexto geográfico diferente, como es la costa de Galicia, hay evidencias para este momento de una erosión continuada de los depósitos litorales, mayoritariamente de origen continental. Estos depósitos ofrecen una gran cantidad de excedente sedimentario en algunos sectores de las rías, como por ejemplo en la de Vigo o la de MurosNoia. Este excedente es lo que favorece la fase de acumulación eólica más reciente. En aquellos sectores donde la dinámica eólica-marina no alcanzó un desarrollo importante, es la formación y expansión de suelos coluviales el rasgo más representativo (COSTA-CASAIS 2001), como también sucede en el interior de Galicia. En el suelo coluvial estudiado por Costa Casais et al. (1994) una datación a $100 \mathrm{~cm}$ de la superficie proporcionó una edad de $530 \pm 80 \mathrm{BP}$ (1281-1511 AD), que se enmarca en el Episodio Cálido Bajomedieval (CosTA-CASAIS 2001).

\subsection{La configuración del paisaje en la Anti- güedad tardía/Alta Edad Media ¿Cambios naturales 0 inducidos?}

El contexto climático es un factor importante para definir la evolución de las formas superficiales, ya que los procesos de erosión-sedimentación se aceleran o reactivan en aquellas fases de mayor o menor intensidad pluviométrica, de fuertes sequías, nevadas, etc. La repercusión o efectividad de los procesos morfogenéticos va a depender del tipo de forma del terreno sobre el que actúan (parte alta de la ladera, fondo del valle, superficie plana, litoral,...) y del agente morfogenético que conduce el proceso. Para entender la dinámica del paisaje en la Antigüedad tardía/Alta Edad Media hay que reconstruirla como un sistema en el que están interactuando un conjunto de variables - suelos y sedimentos, tectónica, condiciones climáticas (sistemas morfogenéticos)- en una escala temporal determinada, donde la acción antrópica va a ser un modelador más de ese paisaje que, dependiendo de su susceptibilidad en el momento de la intervención del ser humano, responderá de una u otra manera. Para ello, es necesario comprender el papel que juegan cada una de estas variables, para así poder analizar los efectos directos e indirectos de su interacción con el paisaje. La combinación e interrelación de estos factores es la que dibuja el paisaje del Noroeste Peninsular entre los siglos V y XI.

En este apartado se realiza una aproximación a la información que aportan las formaciones superficiales, específicamente los suelos coluviales, como archivos paleoambientales, localizados en diferentes puntos geográficos de Galicia (Fig. 2). Estos suelos, son archivos que se pueden utilizar para interpretar los contextos arqueológicos (MANDEL \& BETTIS 2001). Su conocimiento ayuda a revelar la ocupación antrópica y la incidencia de sus actividades en un espacio geográfico determinado. Sus propiedades hace de ellos geoarchivos de gran utilidad para la reconstrucción de los cambios ambientales holocenos (COSTA-CASAIS et al. 2008), donde clima y 
actividad humana han jugado un papel decisivo en su formación. Dada su amplia distribución y potencia, pueden ser cruciales para descifrar y entender las respuestas del ser humano al cambio climático y el impacto provocado por su actividad en el medio ambiente a escala local y regional (LEOPOLD \& VÖLKEL 2007). La reciente aplicación de técnicas de las Ciencias de la Tierra para su análisis en contextos arqueológicos ha generado nuevos niveles de comprensión de las actividades humanas y el uso y la relación con el paisaje a lo largo del tiempo (HERZ \& GARRISON 1998; GOLDBERG \& MACPHAIL 2006; RAPP \& HILL 2006; WILKINSON \& STEVENS 2011).

En Galicia, se documentan suelos coluviales tanto en el interior como en la costa, y en unidades morfológicas diferentes. Las zonas geográficas de las que se disponen más datos a nivel de reconstrucción paleoambiental para el periodo tratado en este trabajo son: el sector de Paredes en Campo Lameiro, antesala de las tierras de la Dorsal Galle- ga; las Sierras Septentrionales (O Xistral) y la costa atlántica gallega. La lectura e identificación de las señales visibles -rasgos geomorfológicos, rasgos morfológicos en los suelos, propiedades físico-químicas, cambios en la composición elemental y mineral, registro geoquímico, composición de la materia orgánica, carbones, polen-, ayudan a realizar una interpretación de los procesos de cambio en el paisaje y sus causas, con la finalidad de obtener una reconstrucción de su transformación. Para ello, se analizan algunas señales conservadas en estos suelos, producto de la actividad humana y las condiciones ambientales, e interpretables en términos de reconstrucción paleoambiental. En este sentido, se hablará de la erosión del suelo, la transformación del bosque, el paisaje del fuego y la acidificación del suelo, aspectos cruciales para entender la evolución y el cambio del paisaje en el Noroeste Peninsular en este momento cultural.

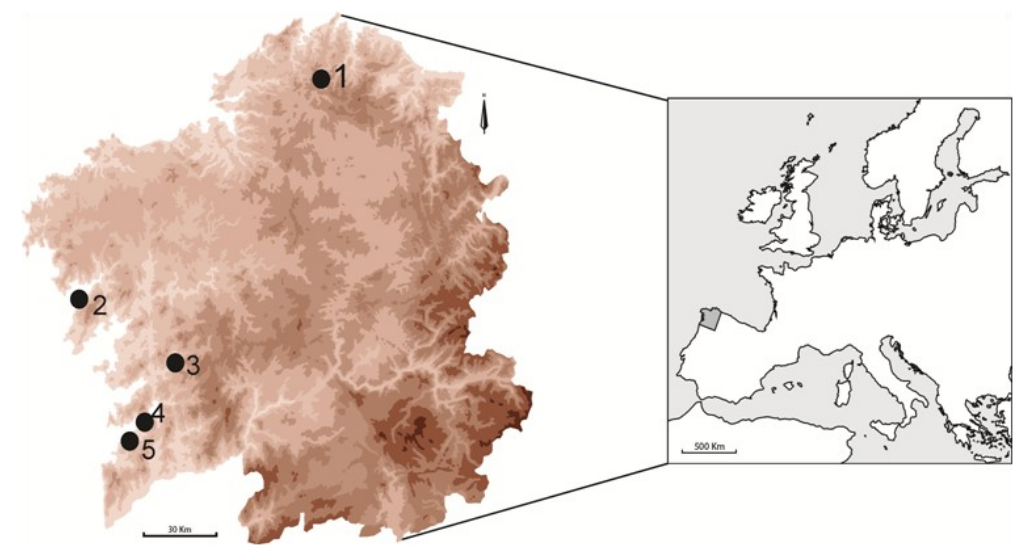

Fig. 2. Localización del área de estudio con indicación de algunos suelos coluviales mencionados en el texto para el NW peninsular (1. Serra do Xistral; 2. Depósito de Caamaño- Porto do Son (A Coruña); 3. Depósitos edafosedimentarios localizados en Monte Paradela- Paredes, Campo Lameiro, Pontevedra); 4. Salinas romanas de Rosalía de Castro-Vigo, Pontevedra); 5. Depóstio de Mirambel-Canido, Vigo, Pontevedra).

Fig. 2. Location of the study area indicating some colluvial soils for the NW Iberian mentioned in the text (1. Serra do Xistral; 2. Depósito de Caamaño-Porto do Son (A Coruña); 3. Pedosedimentary sequences located in Monte Paradela- Paredes, Campo Lameiro (Pontevedra); 4. Salt mines of Rosalía de Castro-Vigo (Pontevedra); 5. Pedo-sedimentary sequence of Mirambel-Canido, Vigo (Pontevedra)

\subsubsection{Inestabilidad en las laderas: erosión y dinámica de los suelos coluviales}

Los suelos coluviales más detalladamente estudiados como registros Holocenos se localizan en Monte Paradela, localizados en Paredes (Campo Lameiro, Pontevedra) (COSTA-CASAIS et al. 2009; KAAL et al. 2008, 2011, 2013; KAAL \& MARTÍNEZ -CORTIZAS 2013). En un sector muy pequeño, que se corresponde con el Parque Arqueológico de Arte Rupestre, se analizaron un número representativo de formaciones coluviales. La toma de muestras de los suelos y los sedimentos se realizó con alta resolución. Estas muestras además han sido contextualizadas cronológicamente con un amplio abanico de dataciones radiocarbónicas. Los suelos mues- treados, que coinciden con los de mayor potencia, se localizan en depresiones y vaguadas, resultado de la erosión de las laderas circundantes (COSTACASAIS et al. 2009; COSTA-CASAIS \& MARTÍNEZCORTIZAS 2013). Con los datos obtenidos MARTíNEZ-CORTIZAS et al. (2009 a, 2013) calcularon las tasas de acumulación/erosión, consideradas como tasas mínimas ya que parte del material transportado pudo haber sido evacuado fuera de la cuenca receptora con posterioridad a su deposición (Fig. 3).

Los resultados obtenidos sugieren la existencia de 16 fases en los últimos 12.000 años, de las cuales dos coinciden con el período cronológico que interesa - el Episodio Frío Altomedieval (4001000 AD). Estas dos son, la penúltima, enmarcada 




Fig. 3. Cambios Holocenos en las tasas de erosion/acumulación, cambio de vegetación, contenido en carbones y reacción del suelo en los suelos coluviales Campo Lameiro, Pontevedra (NW España). El Período Medieval se resalta con color gris (Martínez Cortizas et al. 2009a).

Fig. 3. Holocene changes in soil accumulation/erosion rates, vegetation change, charcoal content and soil reaction in colluvial soils of Campo Lameiro, Pontevedra (NW Spain). The Medieval Time period is highlighted in gray color. (Martínez Cortizas et al. 2009a).

en lo que sería la transición final de la Época Romana y el inicio de la Alta Edad Media (1690-1380 cal $\mathrm{BP} / 310-640 \mathrm{cal} \mathrm{AD})$ y la última que casi se corresponde con la fase final de la Alta Edad Media y la transición a la Baja Edad Media (1190-880 cal BP / 810-1120 cal AD). Los autores señalan que la fase de erosión más intensa se corresponde con esta última. Otros autores, como BENITO el al. (1991) también identifican una fase erosiva significativa entre el 2500-1000 BP (500 BC/-1000 AD). En el sector geográfico de las Sierras Septentrionales de Galicia, MARTÍNEZ-CORTIZAS \& MOARES DOMÍNGUEZ (1995), a partir del estudio de un conjunto de suelos policíclicos, identificaron seis fases erosivas en el Holoceno, de las cuales interesa destacar la señalada entre el 1500-1000 BP (500-1000 AD), que con- cuerda con el momento cultural que nos ocupa LEOPOLD \& VÖLKEL (2007) vinculan la formación de los suelos coluviales con cambios en el clima, particularmente con la precipitación. Precipitaciones extremas pueden inducir eventos catastróficos en el paisaje, provocando la intensidad de la lluvia un gran impacto en la formación y desarrollo de este tipo de suelos. Este hecho apoyaría la idea de relacionar los suelos coluviales datados entre los siglos $\mathrm{V}$ y X, con episodios de intensas lluvias, por lo tanto eventos más húmedos, que provocarían el disparo de la erosión de los suelos en las laderas. Pero este aspecto es difícil de determinar para el Noroeste ibérico, ya que salvo la degradación climática, producida a comienzos de la Alta Edad Media (siglos V y VI) lo que va a caracterizar al resto del período 
son las condiciones climáticas benignas y secas. El ejemplo representativo lo tenemos en Campo Lameiro, donde parece que en algunos períodos secos (1190-880 cal BP / 810-1120 cal AD) la erosión se intensificó, por lo que habría que buscar otras causas diferentes como motor de la formación de los suelos coluviales. En realidad, no toda la precipitación produce procesos erosivos en las laderas, ya que dependerá de otros factores asociados, tales como la intensidad del evento lluvioso, el tipo de unidad geomorfológica que es afectada, la existencia o no de cobertura vegetal, etc. De esta forma, los efectos generados por el agua se suman a otros provocados por las transformaciones antrópicas -talas, quemas, prácticas agrícolas- lo que provoca que los suelos sean más sensibles a los efectos de la erosión. La susceptibilidad de las laderas a los procesos de erosión-acumulación se ven acentuados en eventos climáticos adversos. Quizás el contexto ambiental del Noroeste peninsular en este momento estaría definido por una ocupación antrópica desigual, lo que provocaría una evolución de las formaciones superficiales distinta. Esta antropización diferencial del espacio estaría controlada por el contexto geográfico y la unidad geomorfológica ocupada, lo que genera una respuesta específica de las comunidades locales, para adaptarse al medio, a fin de prevenir la erosión de los suelos y finalmente conseguir aquellos de mejor calidad para sus objetivos inmediatos o ganar nuevos espacios para las prácticas agrícolas, como puede ser mediante la apropiación de tierra, y en muchos casos mediante la construcción de terrazas. Estas formas constructivas comienzan a generalizarse como un elemento más del paisaje a partir del 1500 cal BP (500 cal AD) (BALLESTEROS et al. 2006; MARTÍNEZ CORTIZAS et al. 2009 a; FERRO-VÁZQUEZ et al. 2014).

\subsubsection{El bosque se transforma: uso y efectos del fuego en el paisaje}

Las frecuentes quemas del bosque provocan un cambio en las formaciones vegetales, siendo sustituídas las especies forestales (roble, aliso, abedul, pino) por matorral (brezos, tojos, retamas). Los fuegos, al eliminar la cubierta vegetal, dejan los suelos expuestos a los efectos erosivos, lo que favorece que con la lluvia se generen aguas de escorrentía capaces de movilizar y arrastrar el material. Además, si este está expuesto, las fracciones más finas son más susceptibles de ser erosionadas y transportadas también por la acción eólica. La consecuencia directa es que en áreas geomorfológicas activas el suelo superficial (horizonte A), rico en materia orgánica, puede erosionarse en su totalidad. Este proceso genera la pérdida de fertilidad del suelo, su capacidad de retención de agua, la resistencia a la erosión y su productividad a pesar que las cenizas formadas durante el incendio puedan tener un efecto positivo a corto plazo -encalado y suministro de nutrientes. Los datos antracológicos obtenidos en los suelos coluviales de Paredes (Campo Lameiro, Pontevedra), a partir del análisis de los carbones macroscópicos (>2 mm), relacionan los cambios en la vegetación con los eventos de quema y con los procesos erosión-acumulación en el área (Fig. 3). Los efectos del fuego sobre el bosque caducifolio son manifiestos. El registro de carbones refleja una fuerte caída en la contribución de roble y un incremento en especies de matorral, en particular de brezo, en el 1700 cal BP (300 AD) -final del período romano (KAAL et al. 2013). Este período se puede asimilar con un cambio cultural y climático, siendo la unión de ambos la que generaría el paisaje que se perfilará entre los siglos V y X. Las altas tasas de acumulación de carbones confirmarían que el fuego fue el que estimuló y aceleró la erosión, y transporte de los suelos y sedimentos en las laderas (erosión/ sedimentación), siendo por tanto un agente geomorfológico significativo en su evolución. MARTíNEZ CORTIZAS et al. (2009 a) señalan que los episodios de fuego inducido han destruído el bosque caducifolio y parece que este hecho es sincrónico con la aceleración de la erosión y la formación de suelos coluviales policíclicos, tipo Ranker atlántico, en el NW de España. De hecho, su intenso color negro es muy probable que esté asociado con los residuos de quema ("black carbón") que almacenan, no solamente como fragmentos de carbón macroscópico, si no también como material fino incorporado en los agregados de los suelos (KAAL \& VAN MOURIK 2008; KAAL et al. 2008). La formación de suelos ricos en materia orgánica, a menudo, es el resultado de la acumulación de restos de vegetación quemada debido a incendios inducidos y recurrentes producidos en el bosque a lo largo de miles de años. Las dataciones radiocarbónicas los permiten relacionar con fuegos sucedidos desde el Mesolítico hasta períodos Medievales. Su formación hay que vincularla con la actividad prehistórica, sobre todo con la agricultura, que usaba el fuego como método para obtener la materia orgánica quemada que añadían a los suelos que se cultivaban (SCHMIDT et al. 2002; GEHRT et al. 2002; ECKMEIER et al. 2007).

BLANCO-GONZÁLEZ et al. (2014) señalan que en el Episodio Frío Altomedieval se practicaban estrategias de deforestación en áreas de montaña del Sistema Central de la Península Ibérica - impulsadas principalmente mediante el uso del fuego y que son notables durante el reino Visigodo. Estos autores encuentran en sus registros abundantes hongos coprófilos, que viven en el estiércol, y los relacionan con actividades orientadas a la ganadería y la apertura de nuevos campos de cultivo para cereal. Estas observaciones parecen coincidir con la aplicación de estrategias expansivas agroforestales basadas en la diversificación de los recursos, donde ya se incluyen nuevos cultivos alternativos como la variedad cultivada de olivo (Olea europaea var. europaea) o el castaño (Castanea sativa). En este sentido, también se puede citar que en el sector de Campo Lameiro (Galicia, NW Península Ibérica) 
la actividad agrícola estaría subordinada a los efectos del pastoreo (MARTínEZ CORTIZAS et al. 2009 a).

La incidencia de hambrunas en el Episodio Frío Altomedieval, algunas de ellas mencionadas en fuentes textuales contemporáneas, añaden incertidumbre a las formas de vida agraria en este momento (BlANCO-GONZÁLEZ et al. 2014). Después de la fase inicial de recuperación de los bosques durante los siglos VIII e inicios del X, su aclarado fue continuo a partir del siglo X en el Sistema Central, probablemente relacionado con la explotación estacional de estas áreas. Estos datos pueden ser extrapolados a partir de los altos valores de hongos coprófilos que estos autores encuentran en las muestras analizadas. Fuera del espacio de la sierra, a altitudes más bajas, observan patrones de actividad muy diferentes. El cultivo de cereales está generalizado durante esta fase, ayudado por las condiciones climáticas más suaves que se producen en el Episodio Cálido Bajomedieval. En el lapso de tiempo 1000-1100 AD, los indicadores de incendios y pastoreo son más visibles en algunos registros de polen, como el de La Meseguera (sierra de Peña de Francia), probablemente señalando una mayor presión humana circunstancial e intermitente, en lugar de una mayor permanencia de la población en altitudes medias. La plena integración de los paisajes de alta montaña en el sistema feudal representa la máxima extensión de los pastizales en las tierras altas, mientras que en lugares de baja altitud estos autores citan una especialización marcada en ciertos cultivos - centeno, olivar, castaño.

El estudio polínico de una secuencia sedimentaria localizada en el eje central de la Ría de Vigo (NW península Ibérica) permite a DESPRAT et al. (2003) identificar cambios climáticos en los últimos 3000 años. La información aportada por el registro polínico de los sedimentos ofrece una imagen integrada de la vegetación regional que se relaciona directamente con los parámetros climáticos y el impacto humano. Según esta investigación, los cam- bios importantes en las actividades socioeconómicas de Galicia no fueron sincrónicas con las variaciones regionales en la vegetación, señalando el clima como el responsable para el modelo de reducción del bosque. Las actividades antrópicas tendrían más probabilidad de reducir el bosque desde el 950 cal BC, lo que impediría su recuperación en momentos climáticos de mejoría. Sus resultados muestran que entre el siglo $\mathrm{V}$ y $\mathrm{X}$ hay una pequeña disminución del aliso y el abedul, del mismo modo que el avellano y el carballo. Este último, respecto a Época Romana, sufre un descenso considerable que llega a ser casi de la mitad. El castaño, aunque disminuye respecto al Período Cálido Romano, sigue siendo significativo, destacando los mínimos a inicios del siglo V y VII. En el registro también aparece polen de nogal entre los siglos VI y VII, y de fresno entre el VII y IX. En general, la caída arbórea se corresponde con un mayor aporte de polen de herbáceas, arbustos y cereal. El incremento del cereal es gradual desde el siglo $\mathrm{V}$, mientras que las herbáceas y los arbustos muestran una evolución inversa, manteniendo valores más altos entre los siglos V y IX, las primeras, y más bajos los arbustos, para estas mismas fechas. Estos datos están reflejando la importancia del cultivo de cereal en las laderas de la Ría, siendo la vegetación de herbáceas las más importantes en el episodio frío Altomedieval. Estas variaciones en el polen arbóreo y de plantas arbustivas también son detectadas por MARTíNEz CORTIZAS et al. (2005) a partir de los datos obtenidos en la turbera de Pena da Cadela (PDC) situada en la sierra de O Xistral (Fig. 4). Sus resultados apoyan la interpretación de que las actividades humanas ya desde el Neolítico Final condujeron a una rápida disminución del bosque, debido a la expansión de las tierras de cultivo o pastoreo, pero también inducen otros cambios en los ecosistemas terrestres, tales como la erosión acelerada de los suelos, desafiando la capacidad de las culturas para adaptarse a las nuevas condiciones.

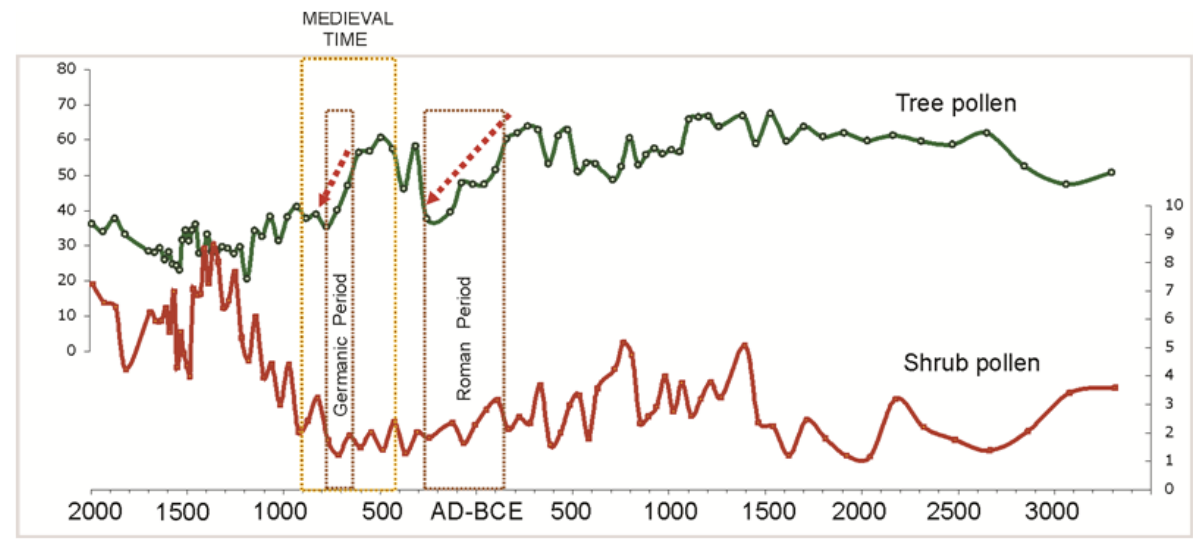

Fig. 4. Cronología de las variaciones en el porcentaje total de polen arboreo y arbustos en el noroeste de España (Modificado de Martínez Cortizas et al. 2005: The Holocene, 15, 5: 698-706.)

Fig. 4. Chronology of the variations in the percentage of total tree pollen and shrub in northwest Spain (Modified from Martínez Cortizas et al. 2005: The Holocene, 15, 5: 698-706.) 


\subsubsection{Efectos directos de los cambios en el pai- saje: Los suelos se acidifican}

La pérdida de suelo por erosión, la redistribución del suelo como recurso en el paisaje y la formación de suelos negros, no son los únicos efectos de la actividad antrópica en épocas prehistóricas e históricas. Hay evidencias de que otras propiedades de los suelos se vieron afectadas por la combinación entre: deforestación, uso del fuego, erosión y modificaciones en la vegetación. Una de estas propiedades es la reacción del suelo $(\mathrm{pH})$ (MARTíNEZ CORTIZAS et al. 2009 a, 2013). Aunque la aceleración de la acidificación se asocia mayoritariamente con la lluvia ácida, vinculada a la Revolución Industrial, las investigaciones llevadas a cabo en los suelos de Paredes (Campo Lameiro) han revelado otra información. El pH del suelo comienza a disminuir desde ca. 6000-5500 cal BP (Fig. 3) hasta hace unos 1000 años, donde parece que se estabilizó (MARTínez Cortizas et al. 2009 a). En los niveles de suelo generados durante períodos de tasas de acumulación alta, los valores de $\mathrm{pH}$ son ligeramente más altos que los niveles que se corresponden con tasas de baja acumulación (MARTíneZ CORTIZAS et al. 2009 a; MARTíneZ CORTIZAS et al. 2013). Este hecho es más visible durante los últimos 3000 años, y se puede relacionar con la entrada de material fresco o escasamente alterado procedente de la erosión de los suelos superficiales localizados en las vertientes. El inicio de esta tendencia es sincrónica con fases de retroceso del bosque, con el incremento de los arbustos en los registros pedoantracológicos, y con un abrupto aumento en el contenido total de carbones en los suelos. Los cambios provocados por la substitución del bosque por una vegetación más acidófila, la ruptura del reciclado de los nutrientes y el incremento de la lixiviación pueden haber sido los motores que provocaron y forzaron la acidificación de los suelos (MARTínEZ CORTIZAS et al. 2009 a; 2013). Resultados similares a los detectados para el sector de Campo Lameiro, se han obtenido en otras áreas geográficas del Noroeste Ibérico, como las Sierras Septentrionales (MARTÍNEZ CORTIZAS et al. 2009 b). Las diferencias vienen determinadas por las tasas de acidificación, que son más altas en las Sierras Septentrionales, debido bien a condiciones climáticas o al material de partida, aunque también la intensificación de las transformaciones antrópicas han jugado un papel importante en esta dirección.

\section{A MODO DE CONCLUSIÓN: EL PAISAJE DEL NOROESTE DE LA PENÍNSULA IBÉRICA ENTRE LOS SIGLOS V Y XI}

Esta investigación propone que clima y actividades humanas han desempeñado un papel trascendental en la transformación de las formaciones superficiales y en la génesis de los suelos coluviales durante la Antigüedad tardía/Alta Edad Media. Los estudios geomorfológicos y sedimentológicos indican que la distribución de las unidades geográficas han sido un factor principal para comprender la formación y el desarrollo de los depósitos coluviales y por tanto para entender los procesos de erosión-sedimentación en laderas y valles. Estos suelos muestran una extensa distribución geográfica y ocupan un amplio abanico temporal, por lo que se han convertido en registros cruciales que contienen información sobre el medio y la actividad antrópica pasada. Por ello se han convertido en registros de gran interés para descifrar y entender las respuestas humanas al cambio climático y el impacto de las actividades antrópicas en el medio tanto a escala local como regional. Estos suelos son geoarchivos que proporcionan información paleoambiental reseñable para el período cronológico de la Antigüedad tardía/Alta Edad Media. En este sentido, cabe destacar que:

- Existe una coincidencia entre las fases de erosión y el período cultural de la Antigüedad tardía/Alta Idade Media.

- Los impactos sobre el medio dependen de la formación geomorfológica ocupada. Dependiendo del contexto climático, a las poblaciones medievales les interesa más ocupar una unidad geomorfológica u otra. Este sentido, BLANCO-GONZÁLEZ et al. (2014) confirman el potencial de las zonas de montaña de gran altitud para evaluar la resiliencia y la vulnerabilidad de los asentamientos de época medieval. Estos autores citan el ejemplo del Sistema Central como un laboratorio sensible para el estudio de las prácticas sociales medievales y su huella en formaciones vegetales. Citan los sectores de montaña como un escenario privilegiado debido a la disponibilidad equilibrada de los recursos para la agricultura, la silvicultura y el pastoreo de las poblaciones medievales que han desarrollado estrategias sucesivas para permanecer en estos ambientes.

- La repercusión directa de las modificaciones en el paisaje se traducen en los cambios geomorfológicos generados por el aumento de las tasas de erosión, coincidentes con la evolución de la cubierta forestal. Se produce una reconfiguración geomorfológica del paisaje. El material se acumula preferentemente en vaguadas y cuencas, frente a unas laderas cada vez más desprovistas de suelo, donde la roca es la protagonista.

- Se dibuja una antropización diferencial del espacio, controlada preferentemente por el contexto geográfico y la unidad geomorfológica ocupada. Esta ocupación diferencial, genera una respuesta específica de las comunidades locales, a fin de prevenir la erosión de los suelos y finalmente conseguir aquellos de mejor calidad para sus objetivos inmediatos o ganar nuevos espacios para las prácticas agrícolas, mediante la apropiación de tierra y la construcción de terrazas agrícolas. Estas, como un elemento constructivo del paisaje, se van a generalizar a partir del $1500 \mathrm{cal}$ BP, con la finalidad de ganar espacio útil para el cultivo. El resultado es la 
configuración de un paisaje dominado por el cultivo, un paisaje agrario, que conoce el funcionamiento de las laderas y las modifica.

- La presencia de carbones y la composición molecular de la materia orgánica indican episodios de incendios frecuentes. Los estudios de polen reflejan una disminución de la cubierta forestal y un aumento de herbáceas y matorral, al mismo tiempo que aumenta el polen de cereal. El cambio en la cubierta forestal es acompañado por la acidificación del suelo.

- Los resultados de los diferentes indicadores proporcionan una explicación múltiple para los cambios ambientales sucedidos en este período cronológico - efecto de las actividades humanas acopladas a los cambios ambientales, que a su vez disparan nuevos cambios. Las situaciones más críticas se suceden de forma sincrónica con transiciones climáticas abruptas, probablemente debido a la combinación de la fragilidad del ambiente y la presión antrópica. Es por ello que el paisaje del NW en la Antigüedad tardía/Alta Edad Media es la consecuencia de esta compleja interacción, dando como resultado la configuración de un Paisaje Cultural.

\section{AGRADECIMIENTOS}

Esta investigación se llevó a cabo en el marco de los proyectos de investigación CPC2014/ 009 y R2014 / 001 financiados por la Xunta de Galicia.

\section{BIBLIOGRAFÍA}

Ballesteros, P.; Criado, F. \& Andrade, J.M. 2006. Formas y fechas de un paisaje agrario de época medieval: A cidade da Cultura en Santiago de Compostela. Arqueología Espacial 26: 193-225.

BARD, E.; RAisbeck, G.; Yiou, F. \& Jouzel, J. 2000. Solar irradiance during the last 1200 years based on cosmogenic nuclides. Tellus 52B: 985-992.

Blanco-GonzÁlez, A.; LóPez-SÁEz, J.A.; Alba, F.; ABEL, D. \& PÉREZ, S. 2014. Medieval landscapes in the Spanish Central System (450-1350): a palaeoenvironmental and historical perspective. Journal of Medieval Iberian Studies. Doi: 10.1080/17546559.2014.925135

Benito E.; Soto, B. \& DíAZ-Fierros, F. 1991. Soil erosion studies in NW Spain. In: M. Sala, J.L.Rubio \& J.M.García-Ruíz (Eds.): Soil erosion studies in Spain. Geoforma Ediciones, 55-74.

Bradley, R.S.; Hughes, M.K. \& DiAZ, H.F. 2003. Climate in Medieval time. Science 302: 404-405.

Brovkin, V.; Claussen, M.; Driesschaert, E.; FICHefet, T.; Kicklighter, T.; Loutre, M.F.; MatThews, H.D.; RAMANKUTTY, N.; SCHAEFFER, M. \& SOKOLOV, A. 2006. Biogeophysical effects of historical land cover changes simulated by six Earth system models of intermediate complexity. Clim. Dyn. 26 (6): 587-600
Büntgen, U.; Frank, D.C.; Nievergelt, D. \& Esper, J 2006. Summer temperature variations in the European Alps, A.D. 755-2004. J. Clim. 19: 5607-5623.

Büntgen, U.; Tegel, W.; NiCOlussi, K.; MCCORMicK, M.; FranK, D.;TROUET, V.; KAPLAN, J.O.; HERZIG, F.; HEUSSNER, K.-U.; WANNER, H.; LUTERBACHER, J. \& ESPER, J. 2011. 2500 years of European climate variability and human susceptibility. Science 331: 578-582.

Costa-CASAIS, M. 2001. Análise sedimentaria e reconstrucción paleoambiental da costa Atlántica de Galicia. Servicio de Publicacións e Intercambio Científico. Universidade de Santiago de Compostela, 236 pp.

Costa-Casais, M. \& Martínez-Cortizas, A. 2013. Dinámica geomorfológica del área de estudio y su relevancia en la transformación del paisaje (Capitulo 4). In F. Criado-Boado, A., Martínez-Cortizas, A. \& García Quintela, M.V. (Eds.) "Petroglifos, paleoambiente y paisaje. Estudios interdisciplinares del arte rupestre de Campo Lameiro (Pontevedra)", Tapa 42. Traballos de Arqueoloxía e Patrimonio (TAPA). Consejo Superior de Investigaciones Científicas. Instituto de Ciencias del Patrimonio (Incipit). CSIC: http:// editorial.csic.es (correo: publ@csic.es): 65-82.

Costa Casais, M.; Martínez Cortizas, A. \& Pérez Alberti, A. 1994. Caracterización de un depósito costero de la Ría de Muros-Noia (La Coruña, Galicia). In: Arnáez-Vadillo, J.M., García-Ruiz, J.M. Gómez Vilar, A (Eds.) Geomorfología en España. Tomo II. S.E.G. Logroño.

Costa-Casais, M.; Martínez Cortizas, A.; KaAL, J.; FERro-VÁzquez, C.\& CRIADO-BOADO, F. 2008 Depósitos coluviales holocenos del NO Peninsular: geoarchivos para la reconstrucción de la dinámica geomorfológica. In: J. Benavente \& F.J. Gracia (Eds.) Trabajos de Geomorfología en España 20062008.SEG, X Reunión Nacional de Geomorfología. Cádiz: 83-86.

Costa-Casais, M.; Martínez-Cortizas, A., Pontevedra -Pombal, X. \& CRIADO-BoAdo, F. 2009. Analysis of landforms in geoarchaeology: Campo Lameiro, NW Iberian Peninsula. Mem. Descr. Carta Geol. D'it. LXXXVII: 3-52.

Costa-Casais, M.; MartíneZ-Cortizas, A.; KaAl, JoeRi.; Caetano Alves, M.I. \& Criado-Boado, F. 2012. Reconstructing Holocene evolution in the archaeological site of Campo Lameiro (NW Spain): An interdisciplinary approach to geoarchaeology. In: A. Campar Almeida, Ana M.S. Bettencourt, D. Moura, Sérgio Monteiro-Rodrigues and Maria Isabel Caetano Alves (Eds.) Environmental changes and human interaction along the Western Atlantic edge. Sersilito-Empresa Grafica, Lda.Coimbra: 51-69.

Corona, C.; Guiot, J.; Edouard, J.L.; Chalié, F.; BÜNTGEN, U.; Nola, P. \& URBINATI, C. 2010. Millennium-long summer temperature variations in the European Alps as reconstructed from tree rings. Clim. Past 6: 379-400.

Delaygue, G. \& Bard, E. 2010. An Antarctic view of Beryllium-10 and solar activity for the past millennium. Clim. Dyn. 36: 2201-2218. Doi:10.1007/ S00382-010-0795-1

Desprat, S.; SÁNChez GoÑI, M.F. \& LOUTRE, M.F. 2003. Revealing climatic variability of the last three millen- 
nia in northwestern Iberia using pollen influx data. Earth and Planetary Science Letters 213: 63-78.

Diaz, H.F. \& STAhle, D.W. 2007. Climate and cultural history in the Americas: an overview. Climatic Change 83: 1-8.

ECKMEIER, E.; Gerlach, R.; Gehrt, E. \& SCHMidT, M.W.I. 2007. Pedogenesis of Chernozems in Central Europe -a review. Geoderma 139: 288-299.

Ferro-VÁzquez, C.; Martínez-Cortizas, A.; NóvoAMuÑOZ. J.C; BALlesteros-ARIAS, P. \& CRIADOBOADO, F. 2014. 1500 years of soil use reconstructed from the chemical properties of a terraced soil sequence. Quaternary International, 346: 28-40.

Font Tullot, I. 1988. Historia del clima en España. Cambios climáticos y sus causas. Ministerio de Transportes, Turismo y Comunicaciones. Instituto Nacional de Meteorología. Madrid. 297 pp.

García, N.O.; Gimeno, L.; DE LA TORRE, L.; NiETO, R. \& AÑEL, J.A. 2005. North Atlantic Oscillation (NAO) and precipitation in Galicia (Spain). Atmósfera: 25-32

GeHRT, E.; Geschwinderd-SCHMIDT, H.; VAN DER BORG, K.; EcKMeIER, E. \& SCHMIDT, M.W.I. 2002. Prehistoric alteration of soil in the Lower Rhine Basin, northwest Germany- archaeological, 14C and geochemical evidence. Geoderma 136: 38-50.

Goldberg , P. \& MAcPhaIL, R.I. 2006. Practical and theoretical geoarchaeology. Blackwell Science Ltd, Blackwell Publishing, USA, UK, Australia, 454 pp.

Goosse, H.; Renssen, H.; Timmermann, A. \& Bradley, R.S. 2005. Internal and forced climate variability during the last millennium: a model-data comparison using ensemble simulations. Quatern. Sci. Rev. 24: $1345-1360$.

Goosse, H.; Arzel, O.; Luterbacher, J.; ManN, M.E.; RENSSEN, H.; RiedWyl, N.; TimmermanN, A.; XoplaKi, E. \& WANNER, H. 2006. The origin of the European "Medieval Warm Period". Climate of the Past. 2: 99-113.

Goosse, H.; Guiot, J.; Mann, M.E.; Dubinkina, S. \& SAllaZ-DAMAZ, Y. 2012. The medieval climate anomaly in Europe: Comparison of the summer and annual mean signals in two reconstructions and in simulations with data assimilation. Global and Planetary Change 84-85: 35-47.

GoudIE, A. 1993. The Human Impact on the Natural Environment, fourth edition. Blackwell Publishers, Oxford, UK. 454 pp.

GRUDD, H. 2008. Torneträsk tree-ring width and density ad 500-2004: a test of climatic sensitivity and a new 1500-year reconstruction of north Fennoscandian summers. Clim. Dyn. 31 843-857.

Guiot, J., Corona, C. \& Escarsel MemBers. 2010. Growing season temperatures in Europe and climate forcings over the past 1400 years. PLOS One 5 (4): 1-15

Herz, N. \& Garrison, Ervan G. 1998. Geological methods for archaeology. Oxford University Press, Oxford, New York. 343 pp.

Hidalgo Cuñarro, J.M. \& Soto Arias, P. 1995. Proyecto arqueológico Villa Romana de Toralla. Memoria de Excavación presentada en la Dirección Xeral de Patrimonio Histórico de Galicia.
Hughes, M.K. \& DiAz, H.F. 1994. Was there a "Medieval Warm Period", and if so, where and when? Climate Change 26: 109-142.

JUNGERIUS, P.D. 1990. The characteristics of dune soils In:Th.W. Bakker; P.D. Jungerius \& J.A. Klipn (Eds.), Dunes of the European Coasts: Geomorphology, hidrology, soils. Catena Supplement 18: 155-162.

KAAL, J. \& VAN MOURIK. 2008. Micromorphological evidence of black carbon in colluvial soils from NW Spain. European Journal of Soil Science 59: 11331140.

KAAL, J. \& MARTíneZ-Cortizas, A. 2013. El uso de la huella molecular de la materia orgánica en geoarqueología (Capítulo 18). In: F. Criado-Boado, A., Martínez-Cortizas, A. \& García Quintela, M.V. (Eds.) "Petroglifos, paleoambiente y paisaje. Estudios interdisciplinares del arte rupestre de Campo Lameiro (Pontevedra)", Tapa 42. Traballos de Arqueoloxía e Patrimonio (TAPA). Capítulo 18: El uso de la huella molecular de la materia orgánica en geoarqueología. Consejo Superior de Investigaciones Científicas. Instituto de Ciencias del Patrimonio (Incipit). CSIC: http://editorial.csic.es (correo: publ@csic.es): 265-272

KaAl, J.; MartínEZ-CORTIZAS, A.; ECKMEIER, E; COSTACasais, M.; SAntos Estévez, M. \& CRIAdo BoADO, F. 2008. Holocene fire history of black colluvial soils revealed by pyrolisis-GC/MS: a case study from Campo Lameiro (NW Spain). Journal of Archaeological Science 35: 2133-2143.

KaAl, J.; CARrión Marco, J.; Asouti, E.; Martín SeiJo, M.; Martínez Cortizas, A.; Costa CASAis, M. \& CRIADO BOADO, F. 2011. Long-term deforestation in NW Spain: linking the Holocene fire history to vegetation change and human activities. Quaternary Science Reviews 30: 161-175.

KaAl, J.; Criado-BoAdo, F.; COSTA-CASAIS; M., LÓPEZSÁEZ, J.A.; LóPEZ-MERINO, L.; Mighall, T.; CARRIÓN, Y.; SILVA SÁNCHEZ N. \& MARTÍNEZCortizas, A. 2013. Prehistoric land use an archaeological hot-spot (the rock art park of Campo Lameiro, NW Spain) inferred from charcoal, synanthropic pollen and non-pollen palynomorph proxies. Journal of Archaeological Science. 40: 1518-1527.

KAPLAN, J.O.; KRUMHARDT, K.M. \& ZimMERMANN, N. 2009. The prehistoric and preindustrial deforestation of Europe. Quatern. Sci. Rev. 28, 3016-3034.

LEOPOLD, M. \& VÖLKEL, J. 2007. Colluvium: definition, differentiation, and possible suitability for reconstructing holocene climate data. quaternary international 162-163: 133-140.

LOWE, J.J. \& WALKER, M.J.C. 2006 Reconstructing Ouaternary Environments. Blackwell online (Oxford, OX, United Kingdom).

MANDEL, R.D. \& BeTtis, E.A. 2001. Use and analysis of soils by archaeologists and geoscientists. In Golderberg, P., Holliday, V.T., Ferring, C.R. (Eds.): Earth Sciences and Archaeology. Kluwer Academic/ Plenum, New York: 173-204 p.

MARTínez CORTIZAS, A. 2000a. La reconstrucción de paleoambientes cuaternarios: Ideas, ejemplos y una síntesis de la evolución del Holoceno en el NW de la Península Ibérica. Estudios do Quaternario 3, APEQ. Lisboa: $31-41$. 
Martínez CORTIZAS, A. 2000b. Archivos geoquímicos para la reconstrucción de los paleoambientes cuaternarios: Ideas y ejemplos. II Jornadas do Quternário da APEQ; Porto, FLUP, 12 - 13 Outubro de 2000.

Martínez-Cortizas, A. \& MoARes Domínguez, C. 1995. Edafología y arqueología. Estudio de yacimientos arqueológicos al aire libre en Galicia. Xunta de Galicia. Santiago de Compostela, 199 p

Martínez Cortizas, A.; Costa CAsais, M. \& MoAres DOMÍNGUEZ, C. 1996. Niveles dunares pleistocenos y holocenos en la costa de Galicia: hipótesis cronológica en base a su grado de edafización. In: A. Pérez Alberti, P. Martini; W.Chesworth, \& A. Martínez Cortizas (Eds.), Dinámica y Evolución de Medios Cuaternarios. Consellería de Cultura, Xunta de Galicia. Santiago de Compostela. pp: 391-404

Martínez Cortizas, A. \& Costa CASAIS, M. 1997. Indicios de variaciones del nivel del mar en la ría de Vigo durante los últimos 3000 años. Gallaecia. 7: 121-135.

Martínez Cortizas, A.; Pontevedra Pombal, X.; GarCÍA RodeJA, E.; NóvoA MuÑoz, J.C. \& SHOTYK, W. 1999a. Mercury in a Sapanish peat bog: Archive of climate change and atmospheric metal deposition. Science, vol.284: 939-942.

Martínez Cortizas, A.; VAlcÁrcel Díaz, M.; PÉreZ Alberti, A.; CAstillo Rodríguez, F. \& Blanco CHAO, R. 1999b. Cambio climático e paleoclimas cuaternarios. In: A. Martínez Cortizas \& A. Pérez Alberti (Coords.) Atlás Climático de Galicia. Xunta de Galicia. Santiago de Compostela. 207 pp.

Martinez-Cortizas, A.; Pontevedra-Pombal, X.; Nóvoa Muñoz, J.C.; Peiteado Varela, E. \& PIÑEIRO REBOLO, R. 2005. Linking changes in atmospheric dust deposition, vegetation change and human activities in northwestern Spain during the last 5300 years The Holocene, 15: 698- 706.

Martínez Cortizas, A.; KaAl, J.; Costa CASAis, M. \& CHEsworth, W. 2009 a. Human activities and Holocene environmental change in NW Spain. In: J. Sánchez Díaz \& S. Asíns (Eds.): Control de la Degradación de los Suelos y Cambio Global. Centro de Investigaciones sobre Desertificacion (CSIC, Universitat de València, Generalitat Valenciana), 193-208.

Martínez Cortizas, A.; Costa Casais, M. \& LópezSÁEZ, A. 2009b. Environmental change in NW Iberia between 700 and 500 cal BC. Quaternary International 200: 77-89.

Martínez Cortizas, A.; Costa-Casais, M.; KaAL, J ; Ferro-VÁzquez, C.; Pontevedra-Pombmal X. \& VIVEEN, W. 2013. De la geoquímica al paisaje: composición elemental de los suelos (Capítulo 16). In F. Criado-Boado, A. Martínez-Cortizas, A. \& M. V. García Quintela (Eds.) "Petroglifos, paleoambiente y paisaje. Estudios interdisciplinares del arte rupestre de Campo Lameiro (Pontevedra)", Tapa 42. Traballos de Arqueoloxía e Patrimonio (TAPA). Consejo Superior de Investigaciones Científicas. Instituto de Ciencias del Patrimonio (Incipit). CSIC: http://editorial.csic.es (correo: publ@csic.es): 239255 .

Muscheler, R.; Joos, F.; Beer, J.; Muller, S.A.; VoN MOOS, M. \& SNOWBALL, I. 2007. Solar activity during the last $1000 \mathrm{yr}$ inferred from radionuclide records. Quatern. Sci. Rev. 26: 82-97.
NAVEIro LÓPEZ, J. \& Soto Arias, P. 1996. Un enclave comercial marítimo tardoromano en el Arenal de Vigo. Actas del Congreso Internacional de urbanismo en el Noroeste, Lugo 1996.

Pongratz, J.; Reick, C.; Raddatz, T. \& Claussen, M. 2008. A reconstruction of global agricultural areas and land cover for the last millennium. Glob. Biogeochem. Cycles 22, GB3018. doi:10.1029/2007GB003153

Pongratz, J.; RadDATZ, T.; ReICK, C.H.; Esch, M. \& Claussen, M. 2009. Radiative forcing from anthropogenic land cover change since AD 800. Geophys. Res. Lett. 36, L02709. doi:10.1029/2008GL036394.

RAPP, G. \& HiLl, C.L. 2006. Geoarchaeology. The EarthScience approach to archaeological interpretation. $2^{\text {nd }}$ editon. Yalle University Press, New Haven and London. 339 pp.

Roberts, N.; Moreno, A.; VALERO-GARCÉS, B.L.; CORELla, J.P.; Jones, M.; AllCOCK, S.; WoODBRIDGE, J.; MORELlÓNM, M.; LUTERBACHER, J.; Xoplaki E, E. \& TÜrkeş, M. 2012. Paleolimnological evidence for an east-west climate see-saw in the Mediterranean since AD 900.Global and Planetary Change 84-85: 23-24.

SCHMIDT, M.W.I.; SKJEMSTAD, J.O. \& JÄGER, C. 2002. Carbon isotope geochemistry and nanomorphology os soil black carbon: black chenozemic soils on Central Europe originate from ancient biomass burning. Global Biogeochemical Cycles 16, 1123. Doi: 10.1029/2002GB001939.

SOTO ARIAS, P. 1994. Dúas actuacións sobre o patrimonio arqueolóxico no concello de Vigo. Olea Europaea, $\mathrm{n}^{\circ}$ 0: 4-7.

TALLÓN-ARMADA, R. 2012. Marco evolutivo dunha explotación salinera de época romana (Vigo, Pontevedra) Memoria de licenciatura. Santiago de Compostela, Xaneiro 2012, 109 pp.

TALlÓN-ARMADA, R., Costa-CASAIS, M.; SCHELlEKENS, J.; TABOADA RODRÍGUEZ, T.; VIVES-FERRÁNDIZ SÁnchez, J.; Ferrer García, C.; ABel SchaAd, D.; LÓPEZ-SÁEZ, J.A.; CARRIÓN MARCO, Y. \& MARTÍNEZ CORTIZAS, A. 2014. Holocene environmental change in Eastern Spain reconstructed through the multiproxy study of a pedo-sedimentary sequence from Les Alcusses (Valencia, Spain). Journal of Archaeological Science. 47: 22-38.

Trouet, V.; Scoure, J.D. \& RaIBLe, C.C. 2012. North Atlantic storminess and Atlantic Meridional Overturing Circulatio during the last Millenium: Reconciling contradictory proxy records of NAO variability. Global and Planetary Change 84-85: 48-55.

WALKINGTON, H. 2010. Soil science applications in archaeological contexts: A review of key challenges. EarthSci. Rev. 103: 122-134.

Wilkinson, K. \& STEVENS, C. 2011. Environmental ar chaeology. Approaches, techniques \& applications The History Press, Great Britain. 320 pp.

WeISS, H. \& BRADLEY, R.S. 2001. What drives societal collapse? Science 291: 609-610. 